\title{
Transverse momentum fluctuations and their correlation with elliptic flow in nuclear collisions
}

\author{
Björn Schenke, ${ }^{1}$ Chun Shen, ${ }^{2,3}$ and Derek Teaney ${ }^{4}$ \\ ${ }^{1}$ Physics Department, Brookhaven National Laboratory, Upton, NY 11973, USA \\ ${ }^{2}$ Department of Physics and Astronomy, Wayne State University, Detroit, MI 48201, USA \\ ${ }^{3}$ RIKEN BNL Research Center, Brookhaven National Laboratory, Upton, NY 11973, USA \\ ${ }^{4}$ Department of Physics and Astronomy, Stony Brook University, Stony Brook, NY 11794, USA
}

\begin{abstract}
We propose observables $v_{0}$ and $v_{0}\left(p_{T}\right)$ which quantify the relative fluctuations in the total transverse momentum at fixed multiplicity. We first study the factorization of the fixed multiplicity momentum dependent two particle correlation function into a product of $v_{0}\left(p_{T}^{a}\right)$ and $v_{0}\left(p_{T}^{b}\right)$ within realistic hydrodynamic simulations. Then we present computations of $v_{0}\left(p_{T}\right)$ for different particle types. We determine the relation between the integrated $v_{0}$ and previously measured observables, and compare results from a hybrid hydrodynamics based model to experimental data. The effects of bulk viscosity and an initial pre-equilibrium stage on the results are quantified. We find that $v_{0}$ is strongly correlated with the initial state entropy per elliptic area, $S / A$. Using this result, we explain how the observed correlations between the elliptic flow and the transverse momentum (both in simulations and experiment) reflect the initial state correlations between $1 / A$ and ellipticity $\varepsilon_{2}$ at fixed multiplicity. We argue that the systematic experimental study of $v_{0}$, with the same sophistication as used for the other $v_{n}$, can contribute significantly to our understanding of quark gluon plasma properties.
\end{abstract}

\section{INTRODUCTION}

One of the goals of the experimental program of heavy ion collisions at the Relativistic Heavy Ion Collider (RHIC) and the Large Hadron Collider (LHC) is to quantify the properties of the Quark Gluon Plasma (QGP) by measuring the response of the nuclear medium to gradients in the energy density. If the system is close enough to equilibrium, this response can be characterized by hydrodynamics. Indeed, hydrodynamic simulations, based on approximate thermal equilibrium, describe an enormous variety of data on the long range azimuthal correlations amongst the produced hadrons [1/5]. Hydrodynamic fits to experimental results on elliptic and triangular flows (as well as other observables) place increasingly precise constraints on the shear and bulk viscosity of the QGP as well as its equation of state [6] 8 .

Most of the experimental and theoretical analyses have focused on the harmonic spectrum. In this case, the azimuthal distribution of particles in each event is expanded in a Fourier series

$$
2 \pi \frac{d N}{d \phi}=N\left[1+\sum_{n=1}^{\infty} V_{n} e^{i n \phi}+\text { c.c. }\right],
$$

where $V_{n}$ is a complex Fourier coefficient and c.c. denotes the complex conjugate. The harmonic spectrum is further binned in transverse momentum 1

$$
2 \pi \frac{d N}{d p d \phi}=\frac{d N}{d p}\left[1+\sum_{n=1}^{\infty} V_{n}(p) e^{i n \phi}+\text { c.c. }\right] .
$$

\footnotetext{
1 Throughout the paper the transverse momentum is denoted $p \equiv$ $p_{T}$, i.e., the $T$ subscript will sometimes be dropped to lighten the notation. In addition we will generally assume boost invariance, so that $N$ is short for $d N / d \eta$ etc.
}

The physical origin of the fluctuations in $V_{n}$ and $V_{n}(p)$ is the following: there are event-by-event fluctuations in the angular distribution of the initial energy density, these fluctuations (and their gradients) drive the collective flow of the system, and this collective flow is ultimately imprinted on the particles and their associated harmonic spectrum, $V_{n}(p)$. In practice, it is the long-range correlations (in pseudo-rapidity) between the produced particles that are measured 3 , 4. The two-particle correlation function yields precise data for the squared Fourier coefficients

$$
v_{n}^{2} \equiv\left\langle\left|V_{n}\right|^{2}\right\rangle,
$$

and the corresponding momentum dependent measures

$$
v_{n}(p) \equiv \frac{\left\langle V_{n}(p) V_{n}^{*}\right\rangle}{\sqrt{\left\langle\left|V_{n}\right|^{2}\right\rangle}} .
$$

Here the angular brackets denotes an average over events. A common scheme to eliminate short range correlations is to require that the correlated pairs are separated by a rapidity gap of order $\Delta \eta>2$. The study of flow gives direct information on the medium response to the energy gradients in the system and the fluctuations in the initial state.

In traditional flow analyses, fluctuations in the transverse momentum, i.e., the event-to-event distribution of $d N / d p$ in Eq. 22, are either not considered or described in somewhat different terms than the $v_{n}$ 9, 10. This distribution is characterized by the two point functions, $\left\langle d N / d p_{1} d N / d p_{2}\right\rangle$. Except in the the principal component analysis of [11, available measurements on momentum fluctuations [9, 10, 12] have neglected the rapidity gap typical in flow analyses, and thus reflect both nonflow correlations and long-range hydrodynamic fluctuations, obscuring the underlying physics. 
In a hydrodynamic picture, the physical origin of the long range momentum correlations is identical to the $v_{n}$ case [13, 14]: there are event-to-event fluctuations in the radius (at fixed multiplicity), these size fluctuations lead to fluctuations in the radial flow, and this flow is in turn imprinted on the momentum spectra of the produced particles. Although momentum fluctuations are perhaps more sensitive to the equation of state and the bulk viscosity of the QCD plasma than harmonic flow [15, 16, momentum fluctuations are not as well studied.

Building on several papers which will be discussed more completely in what follows [17-19, we will define an observable $v_{0}(p)$ (analogous to $v_{n}(p)$ ) that quantifies the momentum fluctuations and is straightforward to measure and interpret. The only minor complication in defining $v_{0}(p)$ is that the multiplicity should be held fixed. The partial covariance method is the right tool for this job [18, and provides a simpler alternative to the "centrality bin width correction" [20] and to principal component analysis [17, 19, 21, 22. The principal component decomposition can be sensitive to non-flow and lacks a compelling measure 23. We hope that measurements of $v_{0}(p)$ for different particles and perhaps jets will become standard. Such measurements can provide complementary information to the harmonic spectrum, shedding light on thermalization in small systems, and the poorly understood "no-man's" land, a region of $p_{T}$ from $2 \ldots 6 \mathrm{GeV}$. Inspired by earlier work, we have found that the fluctuation in the entropy per area is an excellent predictor for the $v_{0}(p)$, and thus measurements of $v_{0}(p)$ can be used to constrain this important property of the initial state. The integrated quantity $v_{0}$ is closely related to early measurements of momentum fluctuations quantified with the nondescript variable, $C_{m}$ [9, 10, 12. In Sect. IIB 1 we will determine the relation between $C_{m}$ and the momentum integrated $v_{0}$. We will also discuss the relation between $v_{0}(p)$ and principal components in Sect. IIB 2 borrowing heavily from the discussion in Ref. [19].

In Sect. IIC 1, we present hydrodynamic simulations with the MUSIC [24 27]+UrQMD [28, 29] hybrid model with IP-Glasma initial conditions 30, 31. We show results for the fixed multiplicity two particle correlation function that measures correlations of the transverse momentum spectrum at different $p_{T}$, and can be used to define $v_{0}\left(p_{T}\right)$ under the assumption of factorization. We show the quality of the factorization in the same section, and compare our results to a simple model from Ref. [19] for the fluctuations of the transverse momentum spectrum. We then present results for $v_{0}\left(p_{T}\right)$ for different particle species and various centralities, and provide quality measurements for different estimators of transverse momentum fluctuations.

In Sect. IID we present comparisons of the hybrid model calculations with existing data on transverse momentum fluctuations, and discuss the effects of bulk viscosity and a pre-equilibrium stage described by the KoMPoST model [32, 33].
Recently, the ATLAS Collaboration, motivated by earlier theoretical work [14, 21], has studied the correlations between $v_{2}$ and $v_{3}$ and the transverse momentum fluctuations [12]. We will compare the hybrid model simulations described above to this recent data in Sect. III. In addition, we will present estimators for this observable, constructed from initial state properties. We find that the entropy per (elliptic) area provides a good initial state predictor for the mean transverse momentum fluctuations $\delta \bar{p}_{T}$, which together with the eccentricity as estimator for the elliptic flow, estimates the $v_{2}^{2}-\bar{p}_{T}$ correlations and their centrality dependence reasonably well. We further show the predictor obtained from a Monte-Carlo Glauber model calculation with high statistics, which also reproduces the characteristic features of the experimental data, reaffirming their geometric origin.

We conclude in Sect. IV and emphasize again the power of detailed $v_{0}\left(p_{T}\right)$ measurements to shed light on quark gluon plasma properties, including thermalization in small systems and the onset of jet quenching.

While finalizing this manuscript a paper by Bozek and Mehrabpour appeared, which partially overlaps with the current work both in methodology and conclusions 34 . We will note the similarities and differences with this paper below.

\section{MOMENTUM FLUCTUATIONS AND $v_{0}$}

For any observable $O$, the event-by-event deviation and variance are defined as

$$
\delta O \equiv O-\langle O\rangle, \quad \sigma_{O}^{2}=\left\langle(\delta O)^{2}\right\rangle .
$$

Similarly, the event-by-event deviation and variance at fixed multiplicity are defined as 18

$$
\begin{aligned}
& \hat{\delta} O \equiv \delta O-\frac{\langle\delta O \delta N\rangle}{\sigma_{N}^{2}} \delta N, \\
& \hat{\sigma}_{O}^{2} \equiv\left\langle(\hat{\delta} O)^{2}\right\rangle=\left\langle(\delta O)^{2}\right\rangle-\frac{\langle\delta O \delta N\rangle^{2}}{\sigma_{N}^{2}} .
\end{aligned}
$$

It follows that the covariance between two observables at fixed multiplicity is

$$
\left\langle\hat{\delta} O_{1} \hat{\delta} O_{2}\right\rangle=\left\langle\delta O_{1} \delta O_{2}\right\rangle-\frac{\left\langle\delta O_{1} \delta N\right\rangle\left\langle\delta O_{2} \delta N\right\rangle}{\sigma_{N}^{2}} .
$$

The subtraction terms are designed to remove the linear correlation between the observable and the multiplicity. Below we will use this so-called "partial correlation method" to analyze the fluctuations of transverse momentum and elliptic flow at fixed multiplicity. This approach was recently used in Ref. 34] for the same purpose.

It is important to emphasize that the primary goal of measuring the variance at fixed multiplicity is to remove trivial centrality fluctuations. While we have used the multiplicity as a centrality measure, other quantities, 
such as the forward transverse energy, can be used. In the ATLAS detector for instance, a natural choice would be the calorimetric measure FCal $\mathcal{E} \equiv \sum E_{T}$, leading to the definition $\hat{\delta} O=\delta O-\langle\delta O \delta \mathcal{E}\rangle \delta \mathcal{E} / \sigma_{\mathcal{E}}^{2}$. This definition would leave the central tracker free to make the correlation measurement in Eq. 8). In a different context, two separate correlation measurements, one at fixed forward $\mathcal{E}$ and one at fixed multiplicity, have already been performed, and the two measurements give nearly identical results, see the right two panels of Fig. 1 from Ref. 35.

The event-by-event spectra and multiplicity $N$ are notated

$$
\mathcal{N}(p) \equiv \frac{d N}{d p}, \quad N=\int_{0}^{\infty} d p \mathcal{N}(p),
$$

We are interested in the fluctuations in the spectra at fixed multiplicity:

$$
\hat{\delta} \mathcal{N}\left(p^{a}\right)=\delta \mathcal{N}\left(p^{a}\right)-\frac{\left\langle\delta \mathcal{N}\left(p^{a}\right) \delta N\right\rangle}{\sigma_{N}^{2}} \delta N
$$

The fluctuations in integrated $p_{T}$ at fixed multiplicity can be used to characterize the spectral fluctuations, and thus we define

$$
\begin{aligned}
P_{T} & \equiv \int_{0}^{\infty} d p p \mathcal{N}(p), \\
\hat{\delta} P_{T} & =\int_{0}^{\infty} d p p \hat{\delta} \mathcal{N}(p) .
\end{aligned}
$$

$\hat{\delta} P_{T}$ is analogous to the $\vec{Q}_{2}$-vector used to define the elliptic flow 36 38. Specifically it is a sum over particles and therefore characterizes the collective response. We define the integrated $v_{0}$ via the variance in the integrated $P_{T}$ at fixed multiplicity

$$
v_{0}^{2}=\frac{\hat{\sigma}_{P_{T}}^{2}}{\left\langle P_{T}\right\rangle^{2}} .
$$

The momentum dependent $v_{0}(p)$ can be defined as:

$$
v_{0}(p)=\frac{1}{\langle\mathcal{N}(p)\rangle} \frac{\left\langle\hat{\delta} \mathcal{N}(p) \hat{\delta} P_{T}\right\rangle}{\hat{\sigma}_{P_{T}}} .
$$

It is evident from Eqs. (10, , 111), and (12) that the integrated $v_{0}$ is determined by $v_{0}(p)$ according to

$$
v_{0} \equiv \frac{\int_{0}^{\infty} d p p\langle\mathcal{N}(p)\rangle v_{0}(p)}{\int_{0}^{\infty} d p p\langle\mathcal{N}(p)\rangle}
$$

We also note that using Eq. (9) it follows that

$$
\int_{0}^{\infty} d p\langle\mathcal{N}(p)\rangle v_{0}(p)=0 .
$$

More generally, if the forward calorimeter is used to define centrality, then the zero on the r.h.s. of Eq. (14) is replaced with the variance of the multiplicity at fixed forward energy, $\sigma_{N}^{2}-\langle\delta N \delta \mathcal{E}\rangle^{2} / \sigma_{\mathcal{E}}^{2}$, which is an interesting quantity in its own right.

It is also interesting to measure $v_{0}(p)$ for a variety of particles, such as $D$ mesons and $J / \psi$, and perhaps jets. We define for species $s$

$$
v_{0, s}(p)=\frac{1}{\left\langle\mathcal{N}_{s}(p)\right\rangle} \frac{\left\langle\hat{\delta} \mathcal{N}_{s}(p) \hat{\delta} P_{T}\right\rangle}{\hat{\sigma}_{P_{T}}},
$$

where the quantities without index $s$ are obtained using all charged hadrons.

In practice, when assuming factorization, $v_{0}(p)$ can be obtained from two particle correlations. Generally, the two particle correlation function is given by

$$
\begin{aligned}
\mathcal{N}_{\Delta}\left(p^{a}, p^{b}\right) & \equiv\left\langle\delta \mathcal{N}\left(p^{a}\right) \delta \mathcal{N}\left(p^{b}\right)\right\rangle \\
& =\left\langle\mathcal{N}\left(p^{a}\right) \mathcal{N}\left(p^{b}\right)\right\rangle-\left\langle\mathcal{N}\left(p^{a}\right)\right\rangle\left\langle\mathcal{N}\left(p^{b}\right)\right\rangle
\end{aligned}
$$

and the correlation function of deviations from the event averaged $\langle\mathcal{N}(p)\rangle$ at fixed multiplicity is

$$
\begin{aligned}
\left\langle\hat{\delta} \mathcal{N}\left(p^{a}\right) \hat{\delta} \mathcal{N}\left(p^{b}\right)\right\rangle & \equiv \\
\left\langle\delta \mathcal{N}\left(p^{a}\right) \delta \mathcal{N}\left(p^{b}\right)\right\rangle & -\frac{\left\langle\delta \mathcal{N}\left(p^{a}\right) \delta N\right\rangle\left\langle\delta \mathcal{N}\left(p^{b}\right) \delta N\right\rangle}{\sigma_{N}^{2}}
\end{aligned}
$$

Now we divide by the mean spectra and define $v_{0}(p)$ from the two particle correlation function

$$
C\left(p^{a}, p^{b}\right)=\frac{\left\langle\hat{\delta} \mathcal{N}\left(p^{a}\right) \hat{\delta} \mathcal{N}\left(p^{b}\right)\right\rangle}{\left\langle\mathcal{N}\left(p^{a}\right)\right\rangle\left\langle\mathcal{N}\left(p^{b}\right)\right\rangle} \approx v_{0}\left(p^{a}\right) v_{0}\left(p^{b}\right)
$$

Here we have assumed that the spectrum of fluctuations factorizes into a product of a function of $p^{a}$ and a function of $p^{b}$. This will need to be checked experimentally. Provided this factorization holds, one can integrate over $p^{b}$ on both sides of Eq. 18, $\int d p^{b} p^{b}\left\langle\mathcal{N}\left(p^{b}\right)\right\rangle \ldots$, and verify the consistence with Eq. 12 .

In practice, to reduce non-flow the two particle correlations probed by $v_{0}\left(p_{T}\right)$ should be computed using a rapidity gap of approximately two units. When a gap is used these formulas should be modified appropriately. We also note that all efficiencies cancel as in the $v_{2}\left(p_{T}\right)$ case, making for a straightforward measurement.

\section{A. Relation of $v_{0}(p)$ to slope fluctuations in a simple model}

Now we determine how $v_{0}(p)$ is related to event-byevent slope modifications of the spectra. To this end, we recall the analysis and model of Ref. [19, which studied principal components of $\mathcal{N}(p)$. In this model the spectrum is written as

$$
\mathcal{N}\left(p_{T}\right)=\left(2 \pi p_{T}\right) N \frac{e^{-2 p_{T} / \bar{p}_{T}}}{\pi \bar{p}_{T}^{2}},
$$


where the parameters $N$ and $\bar{p}_{T}$ fluctuate from event to event. Here $N$ is the event-by-event multiplicity and $\bar{p}_{T}$ is the event-by-event mean $p_{T}$, while the leading factor $\left(2 \pi p_{T}\right)$ is the appropriate measure. Thus, if the parameters $N$ and $\bar{p}_{T}$ fluctuate, the spectrum fluctuates as

$$
\frac{\delta \mathcal{N}\left(p_{T}\right)}{\left\langle\mathcal{N}\left(p_{T}\right)\right\rangle}=\frac{\delta N}{\langle N\rangle}-\frac{2 \delta \bar{p}_{T}}{\left\langle\bar{p}_{T}\right\rangle}+2 \frac{p_{T} \delta \bar{p}_{T}}{\left\langle\bar{p}_{T}\right\rangle^{2}}
$$

Then, unraveling the nested definitions, one finds after a certain amount of algebra

$$
\frac{\left\langle\hat{\delta} \mathcal{N}\left(p_{T}^{a}\right) \hat{\delta} \mathcal{N}\left(p_{T}^{b}\right)\right\rangle}{\left\langle\mathcal{N}\left(p_{T}^{a}\right)\right\rangle\left\langle\mathcal{N}\left(p_{T}^{b}\right)\right\rangle}=\frac{\hat{\sigma}_{\bar{p}_{T}}^{2}}{\left\langle\bar{p}_{T}\right\rangle^{2}}\left(\frac{2 p_{T}^{a}}{\left\langle\bar{p}_{T}\right\rangle}-2\right)\left(\frac{2 p_{T}^{b}}{\left\langle\bar{p}_{T}\right\rangle}-2\right)
$$

where we recall that $\hat{\sigma}_{\bar{p}_{T}}^{2}=\sigma_{\bar{p}_{T}}^{2}-\left\langle\delta \bar{p}_{T} \delta N\right\rangle^{2} / \sigma_{N}^{2}$. It follows for this model that

$$
v_{0}\left(p_{T}\right)=\frac{\hat{\sigma}_{\bar{p}_{T}}}{\left\langle\bar{p}_{T}\right\rangle}\left(\frac{2 p_{T}}{\left\langle\bar{p}_{T}\right\rangle}-2\right) .
$$

As could be anticipated, $v_{0}\left(p_{T}\right)$ describes the fluctuations in the event-by-event mean $\bar{p}_{T}$ at fixed multiplicity and increases linearly with $p_{T}$. We will determine in Sect. IIC1 how well this simple model describes the correlator $C\left(p^{a}, p^{b}\right)$ computed within the IPGlasma+Music+UrQMD hybrid model.

\section{B. Relation of $v_{0}$ to previous measurements}

In this section we compare the integrated $v_{0}$ to other measures of $p_{T}$ fluctuations which exist in the literature.

$$
\text { 1. } C_{m}
$$

The ALICE collaboration defines a variance $C_{m}$ [10], which is slightly different from the definition used by ATLAS [12. In the notation used above one defines

$$
\Delta P_{T}=\delta P_{T}-\frac{\left\langle P_{T}\right\rangle}{\langle N\rangle} \delta N
$$

and ${ }^{2}$

$$
C_{m}=\frac{\left\langle\Delta P_{T} \Delta P_{T}\right\rangle}{\left\langle N^{2}\right\rangle}
$$

Traditionally, because $p_{T}$ fluctuations were considered distinct from flow measurements, no rapidity gap was used.

These measurements suffer from non-flow and unfortunately can not be fairly compared to most hydrodynamic simulations. We will ignore the issue of non-flow in this

\footnotetext{
${ }^{2}$ In practice, self correlations are excluded in these averages.
}

work, and directly compare to the data, while we eagerly await a modern measurement of radial flow fluctuations.

The ALICE Collaboration defines a mean $p_{T}$ measure $M\left(p_{T}\right):$

$$
\begin{aligned}
M\left(p_{T}\right) & \equiv \frac{\left\langle P_{T}\right\rangle}{\langle N\rangle}=\frac{\left\langle\bar{p}_{T} N\right\rangle}{\langle N\rangle}=\left\langle\bar{p}_{T}\right\rangle+\frac{\left\langle\bar{p}_{T} \delta N\right\rangle}{\langle N\rangle}, \\
& \simeq\left\langle\bar{p}_{T}\right\rangle\left(1+\mathcal{O}\left(\frac{1}{\bar{p}_{T}} \frac{d \bar{p}_{T}}{d N} \frac{\sigma_{N}^{2}}{\langle N\rangle}\right)\right) .
\end{aligned}
$$

If the multiplicity bins are very narrow (as was the case for the ALICE measurements [10]), then the fluctuations in $N$ are small and the second term in Eq. 25a can be neglected. In this case $M\left(p_{T}\right)$ is approximately $\left\langle\bar{p}_{T}\right\rangle$. The partial correlation adopted in this work removes any sensitivity to the bin width, and larger bins (with higher statistics) can be used.

With the same approximations, $C_{m}$ is the variance in $\bar{p}_{T}$. Writing $P_{T}=\bar{p}_{T} N$, and substituting $\bar{p}_{T}=\left\langle\bar{p}_{T}\right\rangle+$ $\delta \bar{p}_{T}$ and $N=\langle N\rangle+\delta N$, yields

$$
\begin{aligned}
\frac{\Delta P_{T}}{\left\langle\bar{p}_{T}\right\rangle\langle N\rangle} & =\frac{\delta \bar{p}_{T}}{\left\langle\bar{p}_{T}\right\rangle}-\frac{\left\langle\delta \bar{p}_{T} \delta N\right\rangle}{\left\langle\bar{p}_{T}\right\rangle\langle N\rangle^{2}} \delta N, \\
& \simeq \frac{\delta \bar{p}_{T}}{\left\langle\bar{p}_{T}\right\rangle} .
\end{aligned}
$$

The last term in Eq. 26a is small (see Eq. 25) and surrounding text), leading to the expected result

$$
\frac{C_{m}}{M\left(p_{T}\right)^{2}} \simeq \frac{\sigma_{\bar{p}_{T}}^{2}}{\left\langle\bar{p}_{T}\right\rangle^{2}} .
$$

Finally, the ATLAS Collaboration defines a slightly different quantity from $C_{m}$, called $c_{k}$ which is closely related to $C_{m}$ but studies the variance in $\delta\left(P_{T} / N\right)$. We will not go through the details here, but within the same approximations, $C_{m} \simeq c_{k}$.

The ALICE measure, $C_{m} / M\left(p_{T}\right)^{2}$, is closely related to the $v_{0}$ measure. $v_{0}$ involves $\hat{\delta} P_{T}$ (as opposed to $\delta P_{T}$ ), which subtracts the linear correlation with multiplicity, $\left\langle\delta P_{T} \delta N\right\rangle / \sigma_{N}^{2}$. With similar approximations to Eq. 25, one finds that this correlation is

$$
\frac{\left\langle\delta P_{T} \delta N\right\rangle}{\sigma_{N}^{2}} \simeq \frac{\left\langle\delta \bar{p}_{T} \delta N\right\rangle}{\sigma_{N}^{2}}\langle N\rangle+\left\langle\bar{p}_{T}\right\rangle
$$

and thus

$$
\frac{\hat{\delta} P_{T}}{\left\langle\bar{p}_{T}\right\rangle\langle N\rangle} \simeq \frac{\hat{\delta} \bar{p}_{T}}{\left\langle\bar{p}_{T}\right\rangle} .
$$

This differs from Eq. 26b by the "hats". Thus

$$
v_{0}^{2} \simeq \frac{\hat{\sigma}_{\bar{p}_{T}}^{2}}{\left\langle\bar{p}_{T}\right\rangle^{2}}
$$

and

$$
\frac{C_{m}}{M\left(p_{T}\right)^{2}}=v_{0}^{2}+\frac{\left\langle\delta \bar{p}_{T} \delta N\right\rangle^{2}}{\left\langle\bar{p}_{T}\right\rangle^{2} \sigma_{N}^{2}}
$$



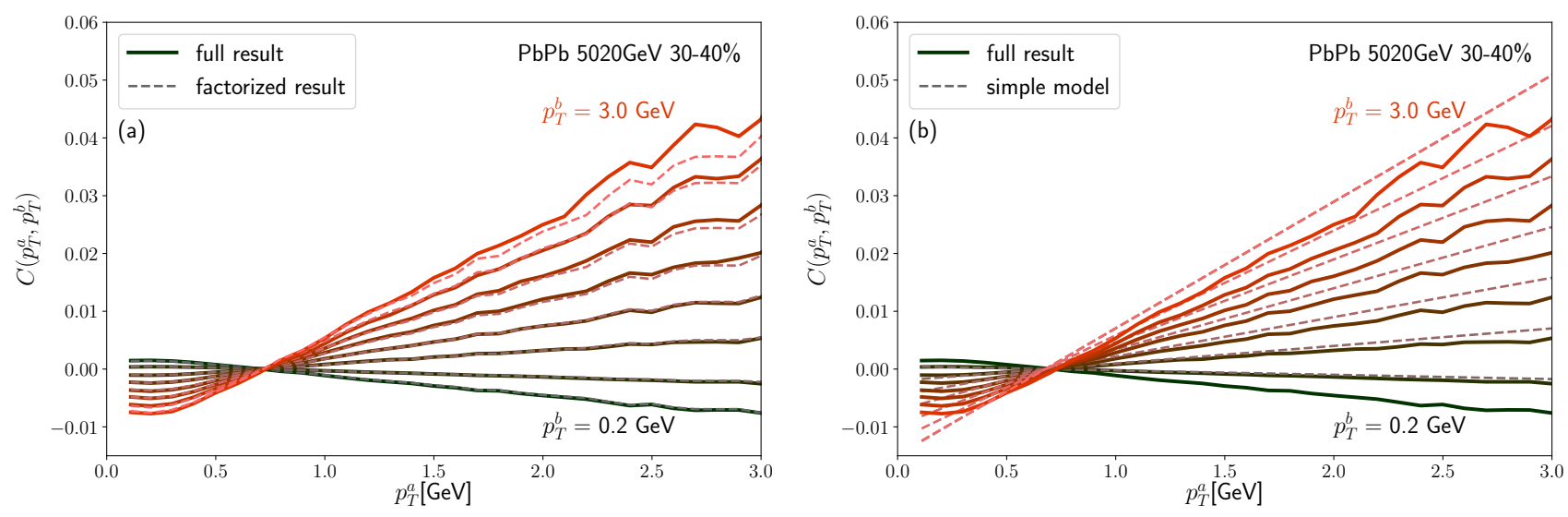

FIG. 1. The correlation function $C\left(p_{T}^{a}, p_{T}^{b}\right)$ from Eq. 18 from the IP-Glasma+MusiC $+\mathrm{UrQMD}$ calculation for $\mathrm{Pb}+\mathrm{Pb}$ collisions at $\sqrt{s}=5020 \mathrm{GeV}$ (solid lines) compared to (a) the factorized form $v_{0}\left(p_{T}^{a}\right) v_{0}\left(p_{T}^{b}\right)$ and (b) the result from the simple model of Ref. [19] given in Eq. 21) (dashed lines). Shown are 8 different $p_{T}^{b}$ values in steps of $0.4 \mathrm{GeV}$.

The last term in Eq. (31) is small if the multiplicity bins are very narrow as was the case with the ALICE measurements.

Measuring both $C_{m}$ and $v_{0}^{2}$ allows access to the physical covariances, $\left\langle\bar{p}_{T}^{2}\right\rangle$ and $\left\langle\delta \bar{p}_{T} \delta N\right\rangle / \sigma_{N}^{2}$. In a principal component analysis of momentum fluctuations (described briefly in the next section), the combination of the leading and subleading principal components also gives access to these variances [19].

\section{Principal components}

Momentum fluctuations have been studied experimentally and theoretically using principal components [11, 17. Briefly, the principal component method breaks up the correlation matrix $\left\langle\mathcal{N}\left(p^{a}\right) \mathcal{N}\left(p^{b}\right)\right\rangle$ into eigen-vectors. The leading eigen-vector predominantly reflects multiplicity (or centrality) fluctuations $\left\langle\delta N^{2}\right\rangle$, while the subleading eigen-vector predominantly reflects momentum fluctuations $\left\langle\left(\delta \bar{p}_{T}\right)^{2}\right\rangle$ [21. Further analysis explained that this description is only approximately true, and in general the leading and subleading eigen-vectors are mixtures of these two contributions [19. In terms of the model discussed in Sect. II A the leading and subleading modes are [19]

$$
\begin{aligned}
& v_{0}^{(1)}\left(p_{T}\right)=\frac{\sigma_{N}}{N}+\left[\frac{-\left(\frac{\sigma_{\bar{p}_{T}}}{\left\langle\bar{p}_{T}\right\rangle}\right)^{2}+2 \frac{\left\langle\delta N \delta \bar{p}_{T}\right\rangle}{\langle N\rangle\left\langle\bar{p}_{T}\right\rangle}}{\frac{\sigma_{N}}{\langle N\rangle}}\right] \frac{p_{T}}{\left\langle\bar{p}_{T}\right\rangle}, \\
& v_{0}^{(2)}\left(p_{T}\right)=-\frac{3}{2} \frac{\sigma_{\bar{p}_{T}}}{\left\langle\bar{p}_{T}\right\rangle}\left(1-\frac{4}{3} \frac{p_{T}}{\left\langle\bar{p}_{T}\right\rangle}\right),
\end{aligned}
$$

which should be compared to Eq. 222. The $v_{0}^{(\alpha)}$ principal components have been measured by the CMS Collaboration [11, and are moderately well described by (ideal) hydrodynamic simulations 19. We believe that the observable $v_{0}\left(p_{T}\right)$ described here can help clarify the discrepancies with hydrodynamic simulations. Further, both theoretically and experimentally, $v_{0}$ is a simpler measure than the corresponding principal components, which often can mix different physics, such as flow and non-flow, in counter-intuitive ways.

\section{Simulations of momentum fluctuations}

\section{IP-Glasma+MUSIC+UrQMD}

In this section we present results for the observables discussed above using simulations of heavy ion collisions with the hybrid framework of IP-Glasma [30, 31, MUSIC [24 26], and UrQMD [28, 29. IP-Glasma provides the initial energy momentum tensor computed from the gluon fields of the incoming nuclei that are described in the Color Glass Condensate framework 39 41. It includes fluctuations of nucleon positions and color charges, which manifest themselves in fluctuations of all components of the energy momentum tensor, leading e.g. to energy density and initial flow velocity fluctuations. MUSIC describes the relativistic hydrodynamic evolution of the initial energy momentum tensor, including shear and bulk viscosity, with all parameters described in [42]. The low energy density regime $\left(e<0.18 \mathrm{GeV} / \mathrm{fm}^{3}\right)$ is described microscopically as an interacting hadron resonance gas using UrQMD. Hadrons for this latter step of the simulation are obtained by first using the CooperFrye [43] procedure to get the off-equilibrium momentum distributions [27, 44, 45] of all particle species and then sampling those distributions [46]. For each hydrodynamic event we run many UrQMD events until the total number of particles per unit rapidity reaches at least 100,000 , and use these to compute particle spectra and flow vectors. This procedure ensures sufficient statistics and eliminates non-flow correlations from UrQMD. 

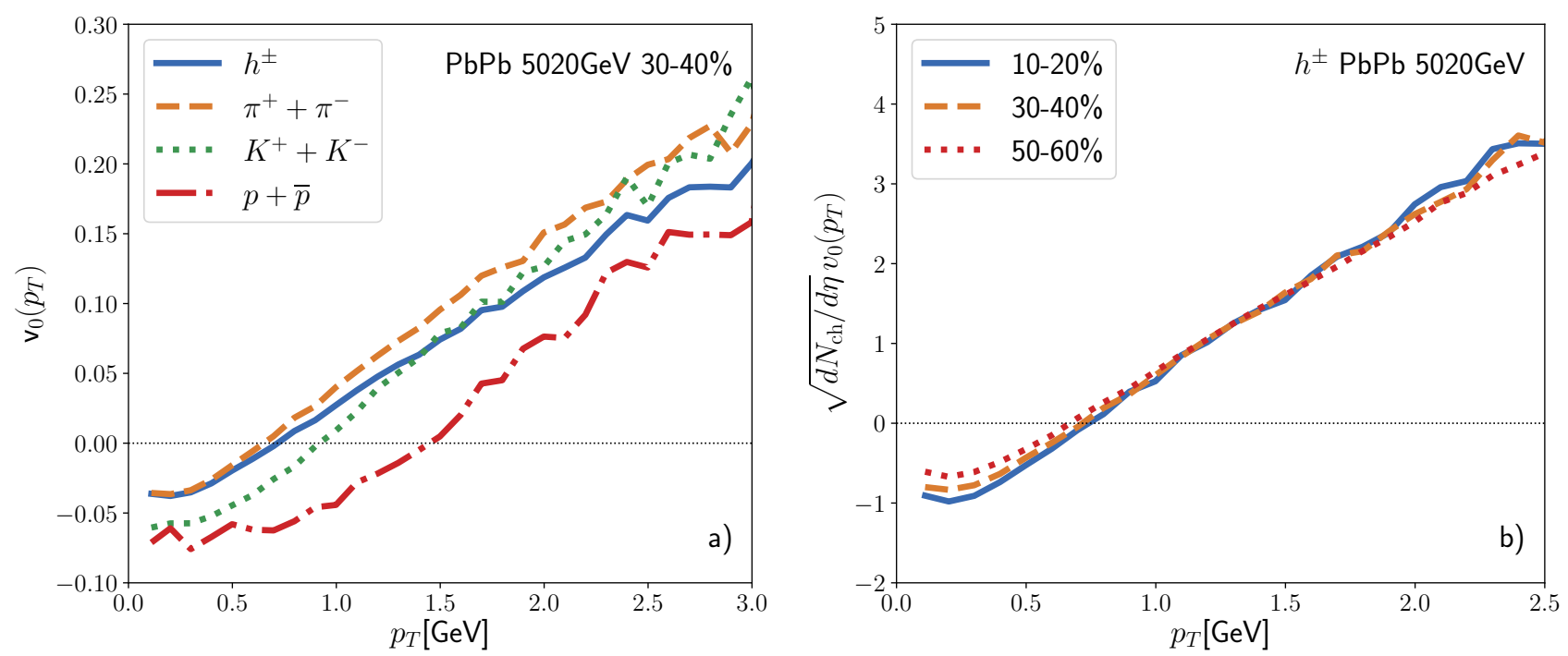

FIG. 2. The momentum dependent $v_{0}\left(p_{T}\right)$ for charged hadrons (solid line) and identified particles (pions (dashed), kaons (dotted), protons (dash-dotted)) in $30-40 \%$ central $\sqrt{s}=5020 \mathrm{GeV} \mathrm{Pb}+\mathrm{Pb}$ collisions (a). Charged hadron $v_{0}\left(p_{T}\right)$ scaled by $\sqrt{d N_{\mathrm{ch}} / d \eta}$ in the respective centrality bin for three different centralities (b).

\section{Results on transverse momentum fluctuations}

We begin by showing the correlator $C\left(p_{T}^{a}, p_{T}^{b}\right)$, defined in Eq. (18), from the hybrid IPGlasma+Music+UrQMD calculation for $\mathrm{Pb}+\mathrm{Pb}$ collisions at $\sqrt{s}=5020 \mathrm{GeV}$ in Fig.1. We show the $p_{T}^{a}$ dependence of the correlator for charged hadrons for 8 different values of $p_{T}^{b}$ using solid lines. To avoid self-correlations, we take the two parts of $C\left(p_{T}^{a}, p_{T}^{b}\right)$ in different rapidity intervals.

In Fig. 1 1a) dashed lines represent the factorized result $v_{0}\left(p_{T}^{a}\right) v_{0}\left(p_{T}^{b}\right)$, using $v_{0}\left(p_{T}\right)$ obtained from the correlator via

$$
v_{0}\left(p^{a}\right)=\frac{\int \mathrm{d} p^{b} p^{b}\left\langle\mathcal{N}\left(p^{b}\right)\right\rangle C\left(p^{a}, p^{b}\right)}{\sqrt{\int \mathrm{d} p^{a} \mathrm{~d} p^{b} p^{a} p^{b}\left\langle\mathcal{N}\left(p^{a}\right)\right\rangle\left\langle\mathcal{N}\left(p^{b}\right)\right\rangle C\left(p^{a}, p^{b}\right)}},
$$

where integrations run from $p_{T}^{a / b}=0$ to a maximum of $p_{T}^{a / b}=4 \mathrm{GeV}$. The result demonstrates that indeed the result factorizes to a good approximation, with significant deviations appearing only for the largest momenta $p_{T}^{a} \sim p_{T}^{b} \sim 3 \mathrm{GeV}$. Similar results were found for other centrality classes.

In Fig.1 1b) we again show the calculation's result for the correlator $C\left(p_{T}^{a}, p_{T}^{b}\right)$ as solid lines, this time compared to the simple model Eq. (21). Agreement between the simple model, which produces linear behavior with $p_{T}^{a}$ and $p_{T}^{b}$ is also rather good, except at the lowest momenta. Also, the model slightly overestimates the full result for most $p_{T}^{b}$ when $p_{T}^{a} \gtrsim 1 \mathrm{GeV}$. We expect all lines to cross at $p_{T}^{a}=\left\langle\bar{p}_{T}\right\rangle$, which is an exact result in the model Eq. (21), and we find the full result to be very close to that.
We show $v_{0}\left(p_{T}\right)$ from the hybrid IPGlasma+Music+UrQMD calculation for $30-40 \%$ central $\mathrm{Pb}+\mathrm{Pb}$ collisions at $\sqrt{s}=5020 \mathrm{GeV}$ in Fig.2 2 ). Besides the results for charged hadrons (solid lines) extracted from the correlator $C\left(p_{T}^{a}, p_{T}^{b}\right)$ discussed above, we also present $v_{0, s}\left(p_{T}\right)$ for identified particles (pions, kaons, protons), defined in Eq. (15). A clear species dependence is visible, with the result for $v_{0}\left(p_{T}\right)$ crossing zero at approximately the mean transverse momentum of the respective particle. The charged hadron $v_{0}\left(p_{T}\right)$ is a weighted average of the individual charged particle $v_{0, s}\left(p_{T}\right)$.

To show the centrality dependence of the signal, and how it scales with $d N_{\mathrm{ch}} / d \eta$, in Fig/2p) we show the charged hadron $v_{0}\left(p_{T}\right)$ for three different centralities, multiplied by the $\sqrt{d N_{\mathrm{ch}} / d \eta}$ in the respective centrality bin. We see that most of the centrality dependence of $v_{0}\left(p_{T}\right)$ is a result of the fluctuations changing as $\left(\sqrt{d N_{\mathrm{ch}} / d \eta}\right)^{-1}$, with small deviations from this scaling at $p_{T}<0.5 \mathrm{GeV}$ caused by the difference in averaged radial flow in different centrality bins.

To determine how strongly the transverse momentum fluctuations correlate with the initial geometry, we introduce predictors for $\hat{\delta} P_{T}$, motivated by earlier work [13, 47, 48]. First, we correlated the fluctuations in the total transverse momentum $\hat{\delta} P_{T}$ with the average entropy density in a given event $[s]$, with the average computed as

$$
[f]=\frac{\int d \mathbf{x}_{\perp} e\left(\mathbf{x}_{\perp}\right) f\left(\mathbf{x}_{\perp}\right)}{\int d \mathbf{x}_{\perp} e\left(\mathbf{x}_{\perp}\right)} .
$$

Here $e\left(\mathbf{x}_{\perp}\right)$ is the initial energy density and $f\left(\mathbf{x}_{\perp}\right)$ is the quantity to be averaged over the transverse plane. Note 
that we recenter such that $\left[\mathbf{x}_{\perp}\right]=(0,0)$.

Next, we correlated $\hat{\delta} P_{T}$ with the fluctuations in total entropy per area, where the circular area is given by $\pi\left[r^{2}\right]$. Including the elliptic deformation in the definition of area can improve this predictor significantly [21, 48]. Thus we define the elliptic area as

$$
A \equiv \pi\left[r^{2}\right] \sqrt{1-\varepsilon_{2}^{2}}=\pi \sqrt{4\left[x^{2}\right]\left[y^{2}\right]},
$$

where $\varepsilon_{2} \equiv\left(\left[y^{2}\right]-\left[x^{2}\right]\right) /\left(\left[x^{2}\right]+\left[y^{2}\right]\right)$, and $x$ and $y$ are measured along the short and long principal axes of the event-by-event ellipse.

To quantify the quality of the predictors, we define the Pearson correlation coefficient

$$
Q_{\xi}=\frac{\left\langle\hat{\delta} P_{T} \hat{\delta} \xi\right\rangle}{\sqrt{\left\langle\hat{\delta} P_{T}^{2}\right\rangle\left\langle\hat{\delta} \xi^{2}\right\rangle}},
$$

where the predictor $\hat{\delta} \xi$ can be defined in various different ways as discussed above. We will use the average event entropy density $\xi=[s]$, the entropy per circular area $\xi=S /\left(\pi\left[r^{2}\right]\right)$, and the entropy per elliptic area $\xi=S / A$. Finally, we use $\xi=\left[r^{2}\right]$ as suggested in the original work [47, which naturally produces a negative correlation, and is expected to be the least efficient estimator.

Results for the different Pearson coefficients $Q_{\xi}$ are shown in Fig.3. We find that for the most central collisions, all predictors work approximately equally well. With decreasing multiplicity, the interaction region becomes more elliptic and taking into account the eccentricity of the area improves the predictor using entropy per area dramatically. The entropy per elliptic area is a simple one term predictor, which combines the most important physics and works well over the full range in centrality. It should be compared with the multi-term predictors of Refs. 21, 34, which separately included the fluctuations in radius, entropy, and squared eccentricities to achieve a similar correlation coefficient.

In rather peripheral collisions, when the eccentricity is large $\varepsilon_{2} \sim 1$ the interaction region typically consists of several disconnected regions and not one single (approximate) ellipse. To increase the mean $p_{T}$ in this regime it is better to have several clustered hot spots leading to a strong hydrodynamic response. Thus, the average entropy density $[s]$ provides a better predictor $p_{T}$ in peripheral bins.

Motivated by Ref. [49, we investigated their preliminary proposal to use the total initial energy (at fixed multiplicity) as a predictor and found it comparable to $[s]$, except in very peripheral events where using $[s]$ works better. We also investigated $E / A$ and found it marginally better than $S / A$.

\section{Comparison with existing data}

We move on to compare our results for transverse momentum fluctuations to existing experimental measurements. In Fig. 4 we show the observable $\sqrt{C_{m}} /\left\langle\bar{p}_{T}\right\rangle$

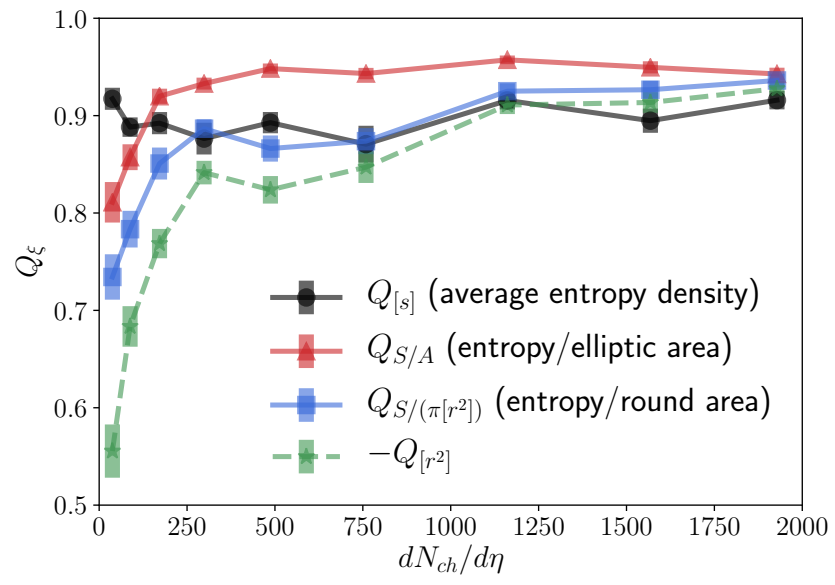

FIG. 3. The Pearson coefficients measuring the quality of different initial state estimators for transverse momentum fluctuations in $\sqrt{s}=5020 \mathrm{GeV} \mathrm{Pb}+\mathrm{Pb}$ collisions.

scaled by $\sqrt{d N_{\mathrm{ch}} / d \eta}$ in $\mathrm{Pb}+\mathrm{Pb}$ collisions at $\sqrt{s}=$ $2760 \mathrm{GeV}$ and compare to the result from the ALICE Collaboration [10. The $p_{T}$ range used to compute the observable is $0.15<p_{T}<2 \mathrm{GeV}$, while the multiplicity is computed over the entire $p_{T}$ range. We show both the regular $\sqrt{C_{m}} /\left\langle\bar{p}_{T}\right\rangle$, which we compute using the approximation in Eq. (27), and the same quantity but with the multiplicity fixed. The latter should compare better to the experimental result, as the multiplicity bins used by the ALICE Collaboration are very narrow. Fixing the multiplicity decreases the fluctuations by removing those resulting from multiplicity fluctuations, and improves agreement with the experimental data. The slope of our result as a function of the multiplicity $d N_{\mathrm{ch}} / d \eta$ is steeper than the experimental data, a trend we will also see in the observable $c_{k}$ measured by the ATLAS Collaboration [12] (see Fig. 7).

The multiplicity dependence of the model follows both from the centrality dependence of the initial state fluctuations of $S / A$, and the hydrodynamic response coefficient $\kappa$

$$
\frac{\hat{\delta} P_{T}}{\left\langle P_{T}\right\rangle}=\kappa \frac{\hat{\delta}(S / A)}{\langle S / A\rangle} .
$$

We would like to disentangle these two contributions. In central collisions the response coefficient is approximately $\kappa_{0} \simeq 0.133$. If $\kappa$ were constant with centrality, the model's $P_{T}$ fluctuations would follow the initial conditions alone

$$
v_{0}=\kappa_{0} \frac{\hat{\sigma}_{S / A}}{\langle S / A\rangle} .
$$

Fig.5 shows this somewhat naive prediction of the IPGlasma model. Interestingly, the initial state estimator given in Eq. (37) reproduces the shape of the experimental measurement somewhat better than the full result of our hybrid framework calculation (also shown in 


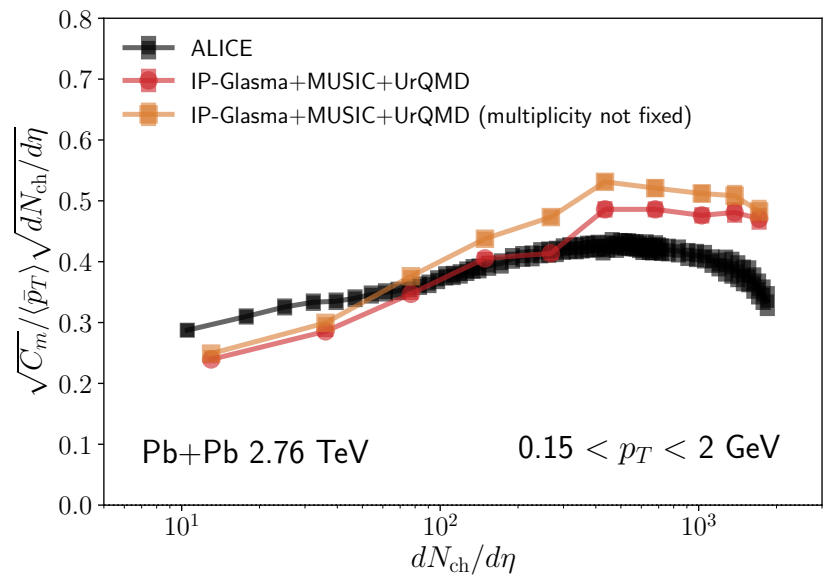

FIG. 4. The observable $\sqrt{C_{m}} /\left\langle\bar{p}_{T}\right\rangle$ scaled by $\sqrt{d N_{\mathrm{ch}} / d \eta}$ in $\mathrm{Pb}+\mathrm{Pb}$ collisions at $\sqrt{s}=2760 \mathrm{GeV}$ compared to experimental data from the ALICE Collaboration [10. Results are computed with (circles) and without (squares) fixing the multiplicity. The simulation result for $\sqrt{C_{m}} /\left\langle\bar{p}_{T}\right\rangle$ at fixed multiplicity (circles) should be compared to the data, and is approximately equal to $v_{0} \sqrt{d N_{\mathrm{ch}} / d \eta}$. See Eq. 31 for the precise relationship between $v_{0}$ and $C_{m}$.

Fig. 57. This might indicate that details of the final state evolution, such as temperature dependent transport coefficients and the freezeout prescription, have not been modeled optimally.

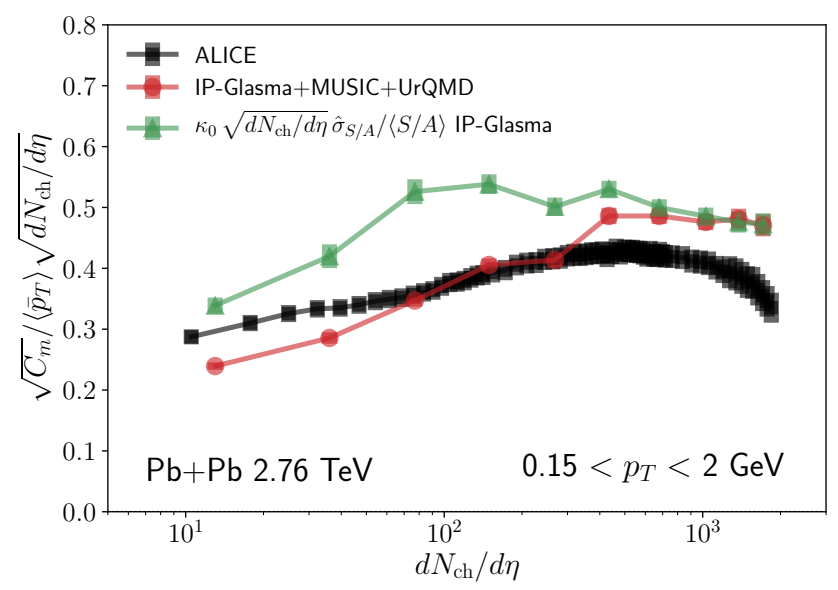

FIG. 5. The observable $\sqrt{C_{m}} /\left\langle\bar{p}_{T}\right\rangle$ at fixed multiplicity scaled by $\sqrt{d N_{\mathrm{ch}} / d \eta}$ in $\mathrm{Pb}+\mathrm{Pb}$ collisions at $\sqrt{s}=2760 \mathrm{GeV}$ (circles) compared to experimental data from the ALICE Collaboration 10]. Also shown are the initial state fluctuations $\kappa_{0} \hat{\sigma}(S / A) /\langle S / A\rangle$ (triangles) taken from the IP-Glasma initial conditions (See Eq. 37)).

To verify whether that is indeed a possibility, we compute $\sqrt{C_{m}} /\left\langle\bar{p}_{T}\right\rangle$ using a simulation with shear viscosity only. As shown in Fig.6, in this case the multiplicity dependence of $\sqrt{C_{m}} /\left\langle\bar{p}_{T}\right\rangle$ is indeed different from the full result including bulk viscosity, which shows that mean-

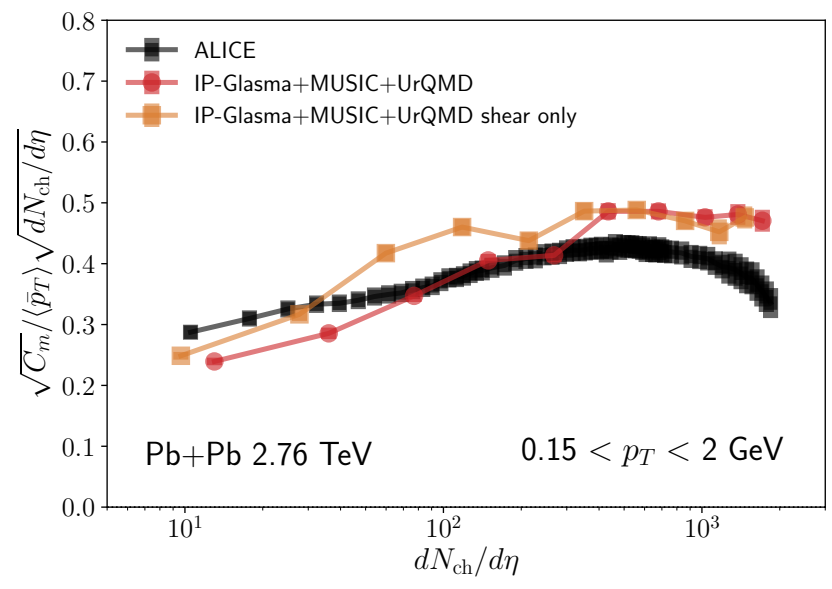

FIG. 6. The observable $\sqrt{C_{m}} /\left\langle\bar{p}_{T}\right\rangle$ at fixed multiplicity scaled by $\sqrt{d N_{\mathrm{ch}} / d \eta}$ in $\mathrm{Pb}+\mathrm{Pb}$ collisions at $\sqrt{s}=2760 \mathrm{GeV}$ (circles) compared to experimental data from the ALICE Collaboration [10, and the result from using no bulk viscosity in the calculation (squares).

$p_{T}$ fluctuations should be considered when constraining transport coefficients in the future. The shear-only result overestimates the fluctuation data in peripheral collisions, and also yields a $\left\langle\bar{p}_{T}\right\rangle$ that is too large [15]. Nevertheless, this exercise demonstrates the sensitivity of mean transverse momentum fluctuations to the choice of transport coefficients, and thus further highlights their importance.

Next, in Fig. 7 we compare to the observable $c_{k}$ as a function of multiplicity, which when measured in narrow multiplicity bins, should be well approximated by $\hat{\sigma}_{\bar{p}_{T}}^{2}$. To be able to compare to the ATLAS measurement 12, we compute $\hat{\sigma}_{\bar{p}_{T}}^{2}$ with $\bar{p}_{T}$ and $\bar{p}_{T}^{2}$ determined in the ranges $0.5<p_{T}<2 \mathrm{GeV}$ and $1<p_{T}<2 \mathrm{GeV}$, with the multiplicity fixed in the interval $0.5<p_{T}<5 \mathrm{GeV}$. In ATLAS, $N_{\mathrm{ch}}$ is determined over 5 units of rapidity, such that we use $N_{\mathrm{ch}}=5 \int d p \mathcal{N}(p)$. Note that we chose to determine $c_{k}$ at fixed multiplicity for this comparison because the multiplicity bins used by ATLAS are significantly narrower than the ones we used.

Agreement with the ATLAS result is reasonable in the range $200<N_{\mathrm{ch}}<2000$, with the experimentally measured $c_{k}$ being underestimated at smaller $N_{\text {ch }}$ and overestimated at larger values. This is the same behavior as we observed for $\sqrt{C_{m}} /\left\langle\bar{p}_{T}\right\rangle$ above. The $p_{T}$-cut dependence is also qualitatively reproduced. We note that using different $p_{T}$ ranges for this observable leads to changing $\left\langle\bar{p}_{T}\right\rangle$ as well as changing $p_{T}$ fluctuations. It would be much more straight forward to simply measure $v_{0}\left(p_{T}\right)$ and the factorization of the $C\left(p_{T}^{a}, p_{T}^{b}\right)$ to gain insight into the $p_{T}$ dependence of fluctuations in the particle spectrum.

The IP-Glasma+MUsiC+UrQMD hybrid framework switches abruptly from the off-equilibrium Yang-Mills system of the IP-Glasma model to viscous hydrodynamics. A more realistic transition between the two 


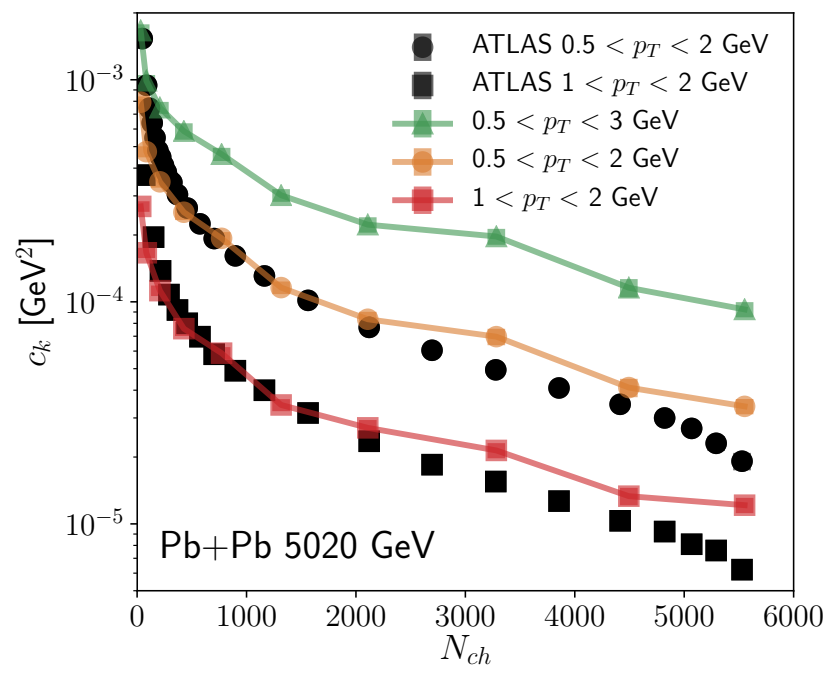

FIG. 7. The observable $c_{k}$, computed here as $c_{k}=\hat{\sigma}_{\bar{p}_{T}}^{2}$ compared to experimental data from the ATLAS Collaboration [12]. We show results for three different $p_{T}$ ranges, two of which were also measured by ATLAS.

stages of the evolution can be obtained by including a pre-equilibrium stage provided by the KoMPoST framework [32, 33. This framework uses nonequilibrium Green's functions from QCD kinetic theory to propagate the IP-Glasma energy-momentum tensor to the hydrodynamic stage, smoothly extrapolating the late stages of the IP-Glasma setup to hydrodynamics where the Music code takes over. Including the preequilibrium stage, the complete model consists of IPGlasma+KoMPoST+Music+UrQMD.

When including a KoMPoST stage, connecting IPGlasma and Music over the interva ${ }^{3}, \tau=0.1-0.8 \mathrm{fm} / c$, we need to modify the used temperature dependent bulk viscosity over entropy density ratio $(\zeta / s)(T)$, to improve the agreement of $\left\langle\bar{p}_{T}\right\rangle$ with experimental data. The preequilibrium dynamics in KoMPoST leads to more radial flow, hence the peak value of $(\zeta / s)(T)$ was increased by $35 \%$ [50].

We present the effect of including a KoMPoST stage in our hybrid model on the measure of mean transverse momentum fluctuations, $\sqrt{C_{m}} /\left\langle\bar{p}_{T}\right\rangle$, at fixed multiplicities, in Fig.88. We find that mean transverse momentum fluctuations, while largely unchanged for the 6 largest multiplicity bins (0-50\% centrality), increase at low multiplicities when using KoMPoST.

\footnotetext{
${ }^{3}$ In the notation of 33. $\tau_{\text {EKT }}=0.1 \mathrm{fm}$ and $\tau_{\text {hydro }}=0.8 \mathrm{fm}$. See Fig. 3 of that reference for an overview.
}

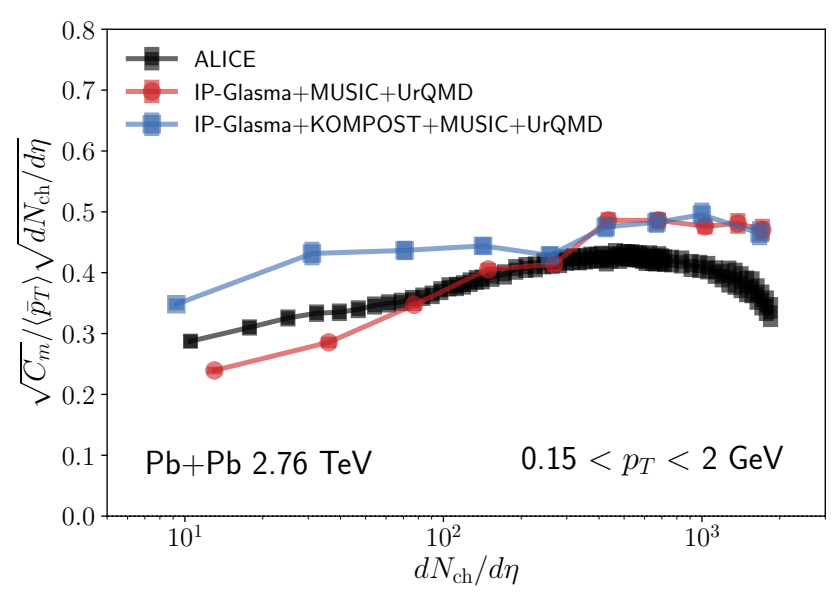

FIG. 8. The observable $\sqrt{C_{m}} /\left\langle\bar{p}_{T}\right\rangle$ at fixed multiplicity scaled by $\sqrt{d N_{\mathrm{ch}} / d \eta}$ in $\mathrm{Pb}+\mathrm{Pb}$ collisions at $\sqrt{s}=2760 \mathrm{GeV}$ (circles) compared to experimental data from the ALICE Collaboration [10, and the result obtained from the simulation including the KoMPoST pre-equilibrium stage (squares).

\section{CORRELATIONS OF ANISOTROPIC FLOW AND MEAN TRANSVERSE MOMENTUM}

In this section, we extend our studies to the correlation between the event-by-event deviation of the squared elliptic flow coefficient and the event-by-event deviation of the mean $p_{T}$, both at fixed multiplicity, using the correlator

$$
\hat{\rho}\left(v_{2}^{2}, \bar{p}_{T}\right)=\frac{\left\langle\hat{\delta} v_{2}^{2} \hat{\delta} \bar{p}_{T}\right\rangle}{\sqrt{\left\langle\left(\hat{\delta} v_{2}^{2}\right)^{2}\right\rangle\left\langle\left(\hat{\delta} \bar{p}_{T}\right)^{2}\right\rangle}} .
$$

The correlation between $v_{2}$ and $p_{T}$ was first studied with principal components in 21, with the prediction that these correlations would drop dramatically in central collisions. The specific correlator in Eq. (38) was developed in Ref. 14] and simplifies the earlier proposal. Further, it was found that $\hat{\rho}\left(v_{2}^{2}, \bar{p}_{T}\right)$ is sensitive to the compactness of the source in proton-nucleus collisions, and thus can be used to differentiate initial state models 14, 34.

To study how well initial state properties determine the final observable, we construct a predictor for $\hat{\rho}\left(v_{2}^{2}, \bar{p}_{T}\right)$. We estimate the fluctuations of the transverse momentum at fixed multiplicity with $\hat{\delta}(S / A)$, which showed the strongest correlation for most centralities, as shown in Fig. 3. We emphasize that since the total initial entropy is tightly correlated with multiplicity (which is held fixed), $\hat{\delta}(S / A)$ is nearly equivalent to $\hat{\delta}(1 / A)$, where $A \equiv \pi\left[r^{2}\right] \sqrt{1-\varepsilon_{2}^{2}}$ is the elliptic area. We further use $\hat{\delta} \varepsilon_{2}$ to estimate the elliptic flow fluctuations, and define the predictor

$$
\hat{\rho}_{\mathrm{est}}\left(v_{2}^{2}, \bar{p}_{T}\right)=\frac{\left\langle\hat{\delta} \varepsilon_{2}^{2} \hat{\delta}(S / A)\right\rangle}{\sqrt{\left\langle\left(\hat{\delta} \varepsilon_{2}^{2}\right)^{2}\right\rangle\left\langle(\hat{\delta}(S / A))^{2}\right\rangle}},
$$


which should track $\hat{\rho}\left(v_{2}^{2}, \bar{p}_{T}\right)$.

\section{A. Results from IP-Glasma+MUSIC+UrQMD}

In Fig.9 we present results from the IPGlasma+MUSIC+UrQMD hybrid model calculation for $\hat{\rho}\left(v_{2}^{2}, \bar{p}_{T}\right)$ in $\sqrt{s}=5020 \mathrm{GeV} \mathrm{Pb}+\mathrm{Pb}$ collisions, along with the predictor $\hat{\rho}_{\text {est }}$ as a function of the ATLAS $N_{\mathrm{ch}}$, and compare to ATLAS experimental data [12.

The expected drop in central collisions is seen in the experimental data, but could not be resolved in the model with current statistics. However, the sign change of the correlator at low multiplicity is qualitatively reproduced. Because the estimator also reproduces this behavior, we conclude that the sign change is a geometrical effect reflecting the correlation between the inverse area $1 / A$ and $\varepsilon_{2}$ at fixed multiplicity. Indeed, in mid-central collisions a smaller area at fixed multiplicity is achieved by fluctuating to larger impact parameters, increasing $\varepsilon_{2}$. The smaller area and correspondingly larger $\varepsilon_{2}$ in this case yields a positive correlation between $\bar{p}_{T}$ and $v_{2}$. By contrast, in quite peripheral collisions a smaller area at fixed multiplicity is achieved by clustering the participants in a single region. This region will be less elliptic through the clustering process. In the clustered case then, the smaller area and correspondingly smaller $\varepsilon_{2}$ yield a negative correlation between $\bar{p}_{T}$ and $v_{2}$.

Because the initial state predictor can approximate the full results very well, it is worth comparing to the predictor obtained from a Monte-Carlo (MC) Glauber model with high statistics. To that effect we generate 4 million minimum bias $\mathrm{Pb}+\mathrm{Pb}$ events at $5020 \mathrm{GeV}$ using an open-source code package SUPERMC 4 , which is a part of the iEBE-VISHNU framework [51. This MC-Glauber model assumed that the system's entropy density is proportional to a mixture of wounded nucleon and binary collision profiles. Multiplicity fluctuations in the local entropy density of individual wounded nucleons and binary collisions were introduced according to a Gamma distribution, which was fitted to the normalized multiplicity distribution in $\mathrm{p}+\mathrm{p}$ collisions assuming Koba-NielsenOlesen (KNO) scaling 52 . We use the lattice equation of state from the hotQCD Collaboration [6] to convert the initial entropy density to energy density, and compute the elliptic area $A$ and eccentricity $\varepsilon_{2}$. All the detailed parameters are listed in Ref. [51. With high statistics, we find that the model estimator decreases in the most central collisions because the eccentricity decreases faster compared to changes in $A$. This estimator reproduces the shape of the $\hat{\rho}$ correlation function very well, although it underestimates the absolute strength by $\sim 20 \%$. The difference between the estimators from MC-Glauber and

\footnotetext{
${ }^{4}$ We use the current version of the SUPERMC code in the github repository, https://github.com/chunshen1987/superMC
}

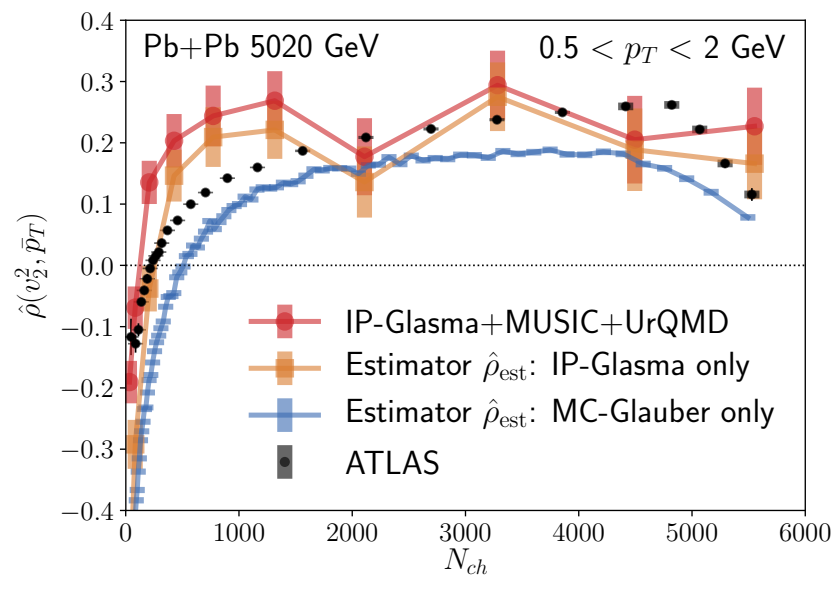

FIG. 9. The correlation measure between $v_{2}^{2}$ and mean transverse momentum $\hat{\rho}\left(v_{2}^{2}, \bar{p}_{T}\right)$ as a function of multiplicity compared to experimental data from the ATLAS Collaboration 12 in $\sqrt{s}=5020 \mathrm{GeV} \mathrm{Pb}+\mathrm{Pb}$ collisions. We further show $\hat{\rho}_{\text {est }}$ from the IP-Glasma (squares) and MC-Glauber (lines) initial state models, using the eccentricity and entropy per elliptic area as predictors for $v_{2}$ and mean $p_{T}$, respectively.

IP-Glasma initial conditions reflects that this observable is sensitive to the details of the initial condition, such as the degree of sub-nucleon fluctuations.

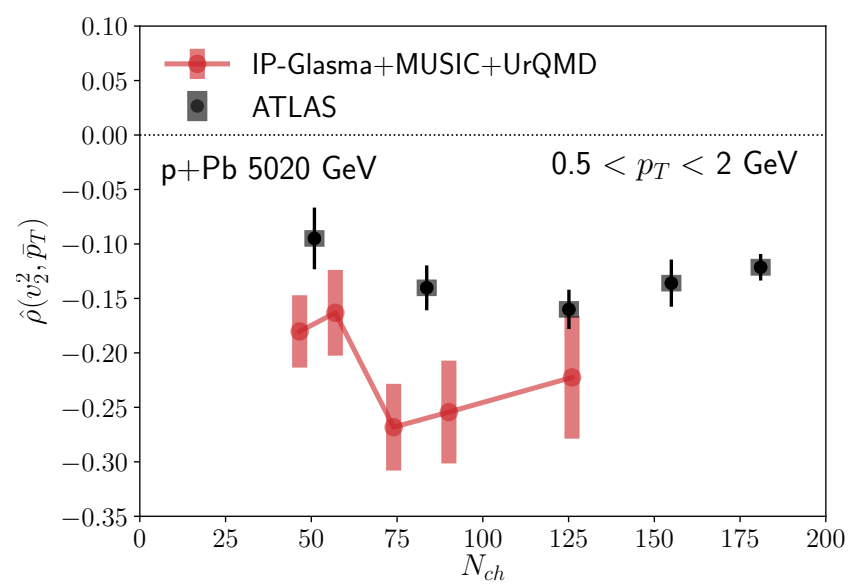

FIG. 10. The correlation measure between $v_{2}^{2}$ and mean transverse momentum $\hat{\rho}\left(v_{2}^{2}, \bar{p}_{T}\right)$ as a function of multiplicity compared to experimental data from the ATLAS Collaboration [12.

We present results for $\hat{\rho}\left(v_{2}^{2}, \bar{p}_{T}\right)$ in $\mathrm{p}+\mathrm{Pb}$ collisions at $\sqrt{s}=5020 \mathrm{GeV}$ in Fig. 10 , and compare to experimental data from the ATLAS Collaboration [12. In this small system, the correlation is negative for all multiplicities, as it is for the most peripheral $\mathrm{Pb}+\mathrm{Pb}$ collisions. While our statistical errors are large, we consistently underestimate the experimental data. The reason for the disagreement could be non-flow contributions to the experimental data or shortcomings in our model, such as the details of the 


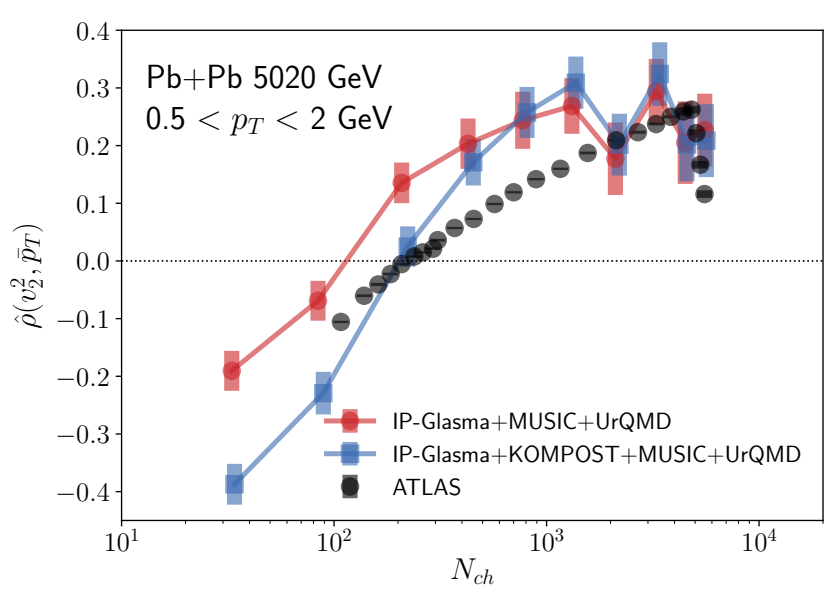

FIG. 11. The correlation measure between $v_{2}^{2}$ and mean transverse momentum $\hat{\rho}\left(v_{2}^{2}, \bar{p}_{T}\right)$ as a function of multiplicity without (circles) and with (squares) a KoMPoST pre-equilibrium stage in the simulation. We compare to experimental data from the ATLAS Collaboration [12] (black circles).

initial state. Indeed, the estimator $\hat{\rho}_{\text {est }}$ (not shown) is even lower than the result for $\hat{\rho}$, with $\hat{\rho}_{\text {est }} \sim-0.5$ to -0.4. In Refs. [14, 34] it was found that a smoother, more Glauber like, initial state leads to a less negative (or even positive) $\hat{\rho}$ correlator.

\section{B. Effect of a pre-equilibrium stage: IP-Glasma+KoMPoST+MUSIC+UrQMD}

As previously done for the mean transverse momentum fluctuation measure $\sqrt{C_{m}} /\left\langle\bar{p}_{T}\right\rangle$, here we study the effect of the pre-equilibrium KoMPoST stage on the $v_{2}^{2}$ $\bar{p}_{T}$ correlator $\hat{\rho}\left(v_{2}^{2}, \bar{p}_{T}\right)$. Fig. 11 shows the comparison of the previously shown IP-Glasma+MUsIC+UrQMD result to the one including a KoMPoST stage and experimental data from the ATLAS Collaboration. Because, again, differences between the simulation with and without KoMPoST are largest at small multiplicities, we use a logarithmic scale for $N_{\mathrm{ch}}$ on the $x$-axis.

For the elliptic flow - mean transverse momentum correlation $\hat{\rho}\left(v_{2}^{2}, \bar{p}_{T}\right)$, the KoMPoST stage leads to a reduction for the ATLAS $N_{\mathrm{ch}} \lesssim 400$. It is expected that the effect of the pre-equilibrium stage is larger for smaller multiplicities, where the hydrodynamic evolution is shorter. Both simulations, with and without KoMPoST, overestimate the experimental data in the range $200 \lesssim N_{\text {ch }} \lesssim 2000$

\section{CONCLUSIONS}

Building on earlier work [18], in Sect. II we introduced an integrated measure of transverse momentum fluctuations at fixed multiplicity, $v_{0} \equiv \hat{\sigma}_{P_{T}} /\left\langle P_{T}\right\rangle$, and the cor- responding differential quantity $v_{0}\left(p_{T}\right)$. Both of these quantities can be measured using two-particle correlations, provided these correlations factorize. The observable $v_{0}\left(p_{T}\right)$ is analogous to the anisotropic flow coefficients $v_{n}\left(p_{T}\right)$ for $n>0$, and its measurement can and should be performed using the standard techniques to eliminate non-flow.

Within a hybrid model consisting of the IP-Glasma initial state, MUsIC hydrodynamics, and UrQMD hadronic cascade, we confirmed the factorization of the twoparticle correlation function into a product of $v_{0}\left(p_{T}^{a}\right)$ and $v_{0}\left(p_{T}^{b}\right)$ (Fig. 1). We further compared the model calculation to the simple model of Ref. [19], that assumes an exponential spectrum whose fluctuations are determined by fluctuations of the mean transverse momentum and the multiplicity.

We further made predictions for $v_{0}\left(p_{T}\right)$ of charged hadrons and identified particles $(\pi, K, p)$, within the hybrid model in $\sqrt{s}=5020 \mathrm{GeV} \mathrm{Pb}+\mathrm{Pb}$ collisions (Fig. 2). The splitting between the hadron species is a telltale signature of radial flow fluctuations. The signal is rather strong, and it should be straight forward to perform the measurement. We further demonstrated that the centrality dependence of charged hadron $v_{0}\left(p_{T}\right)$ is dominated by the scaling of fluctuations with $\left(d N_{\mathrm{ch}} / d \eta\right)^{-1 / 2}$.

The $p_{T}$ integrated $v_{0}$ is closely related to previously measured mean transverse momentum fluctuation observables, at least when measured in narrow bins of multiplicity. The partial correlation method allows for larger multiplicity bins with a corresponding increase in statistics. We discussed the relation of $v_{0}$ to observables previously measured by the ALICE and ATLAS Collaborations in Sect. IIB and presented results for those observables obtained in our hybrid model in Sect. IID. These measurements suffer from non-flow correlations and should be repeated before they can be fairly compared to hydrodynamic simulations.

The magnitude of $v_{0}$ gives direct information on the fluctuations in the initial state. Indeed, we found that the entropy per area is an excellent $v_{0}$ estimator, with a correlation coefficient of $90-95 \%$ over a large part of the centrality range (Fig. $345^{5}$ Thus measurements of $v_{0}$ can tightly constrain the fluctuations in the system size at fixed initial entropy. Interestingly, the estimator gave a somewhat better description of the centrality dependence of the ALICE data on $p_{T}$ fluctuations than the full hybrid model result (Fig. 4 and Fig. 5). This might indicate that final state parameters, such as the temperature dependent bulk viscosity, were not chosen correctly. In fact, we demonstrated that the mean transverse momentum fluctuations are sensitive to the choice of transport parameters (Fig. 6). This lets us conclude that $v_{0}$ should

\footnotetext{
5 For very peripheral systems, the average event entropy density is a better estimator as the interaction area is likely composed of disconnected regions.
} 
be included in every effort to constrain the transport coefficients of QCD using heavy ion collision data.

We further studied the sensitivity of transverse momentum fluctuation measures on the pre-equilibrium stage of the collision by comparing to simulations that transition from the IP-Glasma Yang-Mills stage to hydrodynamics via an off-equilibrium evolution provided by the KoMPoST model (Fig. 8). We found that differences from the usual hybrid model were significant for multiplicities $d N_{\mathrm{ch}} / d \eta \lesssim 200$, demonstrating the power of such observables to access the early time dynamics for small (low multiplicity) systems.

In addition to the analysis of transverse momentum fluctuations, we studied the correlation of elliptic flow with the event-by-event mean transverse momentum within our hybrid model, and compared to experimental data from the ATLAS Collaboration (Fig. 9). We reproduced the qualitative features of the data, including a sign change as a function of multiplicity, and used the entropy per area estimator to explain the simulation results with geometrical reasoning.

The quantitative agreement of our result with the experimental data is not perfect, especially at smaller multiplicities, which could be a result of non-flow in the experimental data, or shortcomings of the model such as the use of less than optimal parameters. Potential problems of non-flow are particularly important for small systems. In $\mathrm{p}+\mathrm{Pb}$ collisions, our model overpredicts the strength of the elliptic flow - mean transverse momentum correlation for most multiplicity bins (Fig. 10). We therefore urge the experiments to reduce the non-flow contributions by employing similar methods for the mean transverse momentum measure as for anisotropic flow coefficients.

To understand the influence of the thermalization stage on the $v_{2}^{2}-\bar{p}_{T}$ correlations, we again compared the simulation results to the experimental data, with and without the KoMPoST thermalization module (Fig. 11). Again we find that the thermalization stage has a significant effect on this observable at small multiplicities, $d N_{\mathrm{ch}} / d \eta \lesssim$
200.

In the future, measurements of $v_{0}\left(p_{T}\right)$ can be used to diagnose the quark gluon plasma in different regimes. For instance, since $v_{0}$ is tightly correlated with the entropy density at fixed system size, $v_{0}\left(p_{T}\right)$ could be measured for jets and others penetrating probes, providing new constraints on the temperature dependence of energy loss. Similarly, measurements of $v_{0}\left(p_{T}\right)$ could clarify the "noman's land", a region of momentum from $2 \ldots 6 \mathrm{GeV}$, which reflects the transition between hydrodynamics to jet-quenching. An upward fluctuation in the entropy (at fixed system size) leads to more hydrodynamic particles at a given $p_{T}$, and simultaneously suppresses the jet contribution through additional energy-loss. Thus, there could be a sign change in $v_{0}\left(p_{T}\right)$ depending on the details of the jet energy loss model. Finally, $v_{0}\left(p_{T}\right)$ can provide important constraints on the initial state fluctuations of the entropy per area in $p+A$ and $p+p$ collisions, and thus help to clarify the thermalization dynamics [53. We hope that the current manuscript can motivate and guide both theorists and experimentalists in these next steps.

\section{ACKNOWLEDGMENTS}

We thank Piotr Bozek, Giuliano Giacalone, and Aleksas Mazeliauskas for useful discussions. BPS is supported under DOE Contract No. de-sc0012704. CS is supported under DOE Contract No. de-sc0013460. DT is supported under DOE Contract No. DE-FG-02-08ER41450. This work is in part supported by the U.S. Department of Energy, Office of Science, Office of Nuclear Physics, within the framework of the Beam Energy Scan Theory (BEST) Topical Collaboration. This research used resources of the National Energy Research Scientific Computing Center, which is supported by the Office of Science of the U.S. Department of Energy under Contract No. DEAC02-05CH11231 and resources of the high performance computing services at Wayne State University.
[1] Derek A. Teaney, "Viscous Hydrodynamics and the Quark Gluon Plasma," in Quark-gluon plasma 4, edited by Rudolph C. Hwa and Xin-Nian Wang (2010) pp. 207266, arXiv:0905.2433 [nucl-th]

[2] Charles Gale, Sangyong Jeon, and Bjoern Schenke, "Hydrodynamic Modeling of Heavy-Ion Collisions," Int. J. of Mod. Phys. A, Vol. 28, 1340011 (2013), arXiv:1301.5893 [nucl-th]

[3] Matthew Luzum and Hannah Petersen, "Initial State Fluctuations and Final State Correlations in Relativistic Heavy-Ion Collisions," J. Phys. G41, 063102 (2014), arXiv:1312.5503 [nucl-th].

[4] Ulrich Heinz and Raimond Snellings, "Collective flow and viscosity in relativistic heavy-ion collisions," Ann. Rev. Nucl. Part. Sci. 63, 123-151 (2013), arXiv:1301.2826 [nucl-th]
[5] Sangyong Jeon and Ulrich Heinz, "Introduction to Hydrodynamics," Int. J. Mod. Phys. E24, 1530010 (2015), arXiv:1503.03931 [hep-ph].

[6] A. Bazavov et al. (HotQCD), "Equation of state in (2+1)-flavor QCD," Phys. Rev. D90, 094503 (2014), arXiv:1407.6387 [hep-lat]

[7] J. Scott Moreland, Jonah E. Bernhard, and Steffen A. Bass, "Estimating initial state and quark-gluon plasma medium properties using a hybrid model with nucleon substructure calibrated to $p-\mathrm{Pb}$ and $\mathrm{Pb}-\mathrm{Pb}$ collisions at $\sqrt{s_{\mathrm{NN}}}=5.02 \mathrm{TeV}, "$ Phys. Rev. C101, 024911 (2020), arXiv:1808.02106 [nucl-th]

[8] Fernando G. Gardim, Giuliano Giacalone, and JeanYves Ollitrault, "Measuring the speed of sound of the quark-gluon plasma in ultracentral nucleus-nucleus collisions," (2019), arXiv:1909.11609 [nucl-th] 
[9] J. Adams et al. (STAR Collaboration), "Incident energy dependence of $p_{t}$ correlations at relativistic energies," Phys. Rev. C 72, 044902 (2005).

[10] Betty Bezverkhny Abelev et al. (ALICE), "Event-byevent mean $p_{\mathbf{T}}$ fluctuations in $\mathrm{pp}$ and $\mathrm{Pb}-\mathrm{Pb}$ collisions at the LHC," Eur. Phys. J. C74, 3077 (2014), arXiv:1407.5530 [nucl-ex].

[11] A. M. Sirunyan et al. (CMS), "Principal-component analysis of two-particle azimuthal correlations in $\mathrm{PbPb}$ and pPb collisions at CMS," Phys. Rev. C96, 064902 (2017), arXiv:1708.07113 [nucl-ex].

[12] Georges Aad et al. (ATLAS), "Measurement of flow harmonics correlations with mean transverse momentum in lead-lead and proton-lead collisions at $\sqrt{s_{N N}}=5.02$ TeV with the ATLAS detector," Eur. Phys. J. C79, 985 (2019), arXiv:1907.05176 [nucl-ex]

[13] Piotr Bozek and Wojciech Broniowski, "Transversemomentum fluctuations in relativistic heavy-ion collisions from event-by-event viscous hydrodynamics," Phys. Rev. C85, 044910 (2012), arXiv:1203.1810 [nucl-th]

[14] Piotr Bozek, "Transverse-momentum-flow correlations in relativistic heavy-ion collisions," Phys. Rev. C93, 044908 (2016), arXiv:1601.04513 [nucl-th]

[15] S. Ryu, J. F. Paquet, C. Shen, G. S. Denicol, B. Schenke, S. Jeon, and C. Gale, "Importance of the Bulk Viscosity of QCD in Ultrarelativistic Heavy-Ion Collisions," Phys. Rev. Lett. 115, 132301 (2015), arXiv:1502.01675 [nuclth],

[16] Fernando G. Gardim, Giuliano Giacalone, Matthew Luzum, and Jean-Yves Ollitrault, "Revealing QCD thermodynamics in ultrarelativistic nuclear collisions," (2019), arXiv:1908.09728 [nucl-th]

[17] Rajeev S. Bhalerao, Jean-Yves Ollitrault, Subrata Pal, and Derek Teaney, "Principal component analysis of event-by-event fluctuations," Phys. Rev. Lett. 114, 152301 (2015), arXiv:1410.7739 [nucl-th]

[18] Adam Olszewski and Wojciech Broniowski, "Partial correlation analysis method in ultra-relativistic heavy-ion collisions," Phys. Rev. C96, 054903 (2017), arXiv:1706.02862 [nucl-th]

[19] Fernando G. Gardim, Frédérique Grassi, Pedro Ishida, Matthew Luzum, and Jean-Yves Ollitrault, " $p_{T^{-}}$ dependent particle number fluctuations from principalcomponent analyses in hydrodynamic simulations of heavy-ion collisions," Phys. Rev. C100, 054905 (2019), arXiv:1906.03045 [nucl-th].

[20] see for example, B. I. Abelev et al. (STAR Collaboration), "Growth of long range forward-backward multiplicity correlations with centrality in $\mathrm{Au}+\mathrm{Au}$ collisions at $\sqrt{s_{N N}}=200 \mathrm{GeV}$," Phys. Rev. Lett. 103, 172301 (2009).

[21] Aleksas Mazeliauskas and Derek Teaney, "Fluctuations of harmonic and radial flow in heavy ion collisions with principal components," Phys. Rev. C93, 024913 (2016). arXiv:1509.07492 [nucl-th].

[22] Mauricio Hippert, David Dobrigkeit Chinellato, Matthew Luzum, Jorge Noronha, Tiago Nunes da Silva, and Jun Takahashi, "Measuring Momentum-Dependent Flow Fluctuations in Heavy-Ion Collisions," Phys. Rev. C101, 034903 (2020), arXiv:1906.08915 [nucl-th]

[23] Ziming Liu, Arabinda Behera, Huichao Song, and Jiangyong Jia, "Robustness of principal component analysis on harmonic flow in heavy ion collisions," (2020), arXiv:2002.06061 [nucl-ex].
[24] Bjoern Schenke, Sangyong Jeon, and Charles Gale, " $(3+1) D$ hydrodynamic simulation of relativistic heavyion collisions," Phys. Rev. C82, 014903 (2010), arXiv:1004.1408 [hep-ph]

[25] Bjorn Schenke, Sangyong Jeon, and Charles Gale, "Elliptic and triangular flow in event-by-event $(3+1) \mathrm{D}$ viscous hydrodynamics," Phys. Rev. Lett. 106, 042301 (2011), arXiv:1009.3244 [hep-ph].

[26] Bjorn Schenke, Sangyong Jeon, and Charles Gale, "Higher flow harmonics from $(3+1)$ D event-by-event viscous hydrodynamics," Phys. Rev. C85, 024901 (2012), arXiv:1109.6289 [hep-ph].

[27] Jean-François Paquet, Chun Shen, Gabriel S. Denicol, Matthew Luzum, Björn Schenke, Sangyong Jeon, and Charles Gale, "Production of photons in relativistic heavy-ion collisions," Phys. Rev. C93, 044906 (2016) arXiv:1509.06738 [hep-ph].

[28] S. A. Bass et al., "Microscopic models for ultrarelativistic heavy ion collisions," Prog. Part. Nucl. Phys. 41, 255-369 (1998), [Prog. Part. Nucl. Phys.41,225(1998)], arXiv:nucl-th/9803035 [nucl-th].

[29] M. Bleicher et al., "Relativistic hadron hadron collisions in the ultrarelativistic quantum molecular dynamics model," J. Phys. G25, 1859-1896 (1999), arXiv:hepph/9909407 [hep-ph]

[30] Bjoern Schenke, Prithwish Tribedy, and Raju Venugopalan, "Fluctuating Glasma initial conditions and flow in heavy ion collisions," Phys. Rev. Lett. 108, 252301 (2012), arXiv:1202.6646 [nucl-th],

[31] Bjoern Schenke, Prithwish Tribedy, and Raju Venugopalan, "Event-by-event gluon multiplicity, energy density, and eccentricities in ultrarelativistic heavy-ion collisions," Phys. Rev. C86, 034908 (2012), arXiv:1206.6805 [hep-ph]

[32] Aleksi Kurkela, Aleksas Mazeliauskas, Jean-François Paquet, Sören Schlichting, and Derek Teaney, "Effective kinetic description of event-by-event pre-equilibrium dynamics in high-energy heavy-ion collisions," Phys. Rev. C99, 034910 (2019), arXiv:1805.00961 [hep-ph].

[33] Aleksi Kurkela, Aleksas Mazeliauskas, Jean-François Paquet, Sören Schlichting, and Derek Teaney, "Matching the Nonequilibrium Initial Stage of Heavy Ion Collisions to Hydrodynamics with QCD Kinetic Theory," Phys. Rev. Lett. 122, 122302 (2019), arXiv:1805.01604 [hep$\mathrm{ph}]$

[34] Piotr Bozek and Hadi Mehrabpour, "Correlation coefficient between harmonic and transverse flow in heavy-ion collisions," (2020), arXiv:2002.08832 [nucl-th].

[35] The ATLAS collaboration (ATLAS), "Measurement of two-particle pseudorapidity correlations in lead-lead collisions at $\sqrt{s_{N N}}=2.76 \mathrm{TeV}$ with the ATLAS detector," ATLAS-CONF-2015-020 (2015).

[36] Jean-Yves Ollitrault, "Anisotropy as a signature of transverse collective flow," Phys. Rev. D46, 229-245 (1992).

[37] S. Voloshin and Y. Zhang, "Flow study in relativistic nuclear collisions by Fourier expansion of Azimuthal particle distributions," Z. Phys. C70, 665-672 (1996), arXiv:hep-ph/9407282 [hep-ph].

[38] J. Barrette et al. (E877), "Observation of anisotropic event shapes and transverse flow in $\mathrm{Au}+\mathrm{Au}$ collisions at AGS energy," Phys. Rev. Lett. 73, 2532-2535 (1994), arXiv:hep-ex/9405003 [hep-ex].

[39] Larry D. McLerran and Raju Venugopalan, "Computing quark and gluon distribution functions for very large 
nuclei," Phys. Rev. D49, 2233-2241 (1994)

[40] Larry D. McLerran and Raju Venugopalan, "Gluon distribution functions for very large nuclei at small transverse momentum," Phys. Rev. D49, 3352-3355 (1994)

[41] Edmond Iancu and Raju Venugopalan, "The color glass condensate and high energy scattering in QCD," in Quark gluon plasma, edited by R.C. Hwa and X. N. Wang (World Scientific, 2003) hep-ph/0303204

[42] Björn Schenke, Chun Shen, and Prithwish Tribedy, "Multi-particle and charge-dependent azimuthal correlations in heavy-ion collisions at the Relativistic Heavy-Ion Collider," Phys. Rev. C99, 044908 (2019) arXiv:1901.04378 [nucl-th].

[43] Fred Cooper and Graham Frye, Phys. Rev. D10, 186 (1974)

[44] Kevin Dusling, Guy D. Moore, and Derek Teaney, "Radiative energy loss and v2 spectra for viscous hydrodynamics," Phys. Rev. C81, 034907 (2010)

[45] Piotr Bozek, "Bulk and shear viscosities of matter created in relativistic heavy-ion collisions," Phys. Rev. C81, 034909 (2010), arXiv:0911.2397 [nucl-th]

[46] https://github.com/chunshen1987/iSS

[47] Wojciech Broniowski, Mikolaj Chojnacki, and Lukasz Obara, "Size fluctuations of the initial source and the event-by-event transverse momentum fluctuations in relativistic heavy-ion collisions," Phys. Rev. C80, 051902 (2009), arXiv:0907.3216 [nucl-th]
[48] Piotr Bożek and Wojciech Broniowski, "Transverse momentum fluctuations in ultrarelativistic $\mathrm{Pb}+\mathrm{Pb}$ and $\mathrm{p}+$ Pb collisions with "wounded" quarks," Phys. Rev. C96, 014904 (2017), arXiv:1701.09105 [nucl-th]

[49] Fernando G. Gardim, Giuliano Giacalone, Matthew Luzum, and Jean-Yves Ollitrault, "Effects of initial state fluctuations on the mean transverse momentum," (2020), arXiv:2002.07008 [nucl-th]

[50] Charles Gale, Jean-François Paquet, Björn Schenke, and Chun Shen, "Probing Early-Time Dynamics and Quark-Gluon Plasma Transport Properties with Photons and Hadrons," in 28th International Conference on Ultrarelativistic Nucleus-Nucleus Collisions (Quark Matter 2019) Wuhan, China, November 4-9, 2019 (2020) arXiv:2002.05191 [hep-ph].

[51] Chun Shen, Zhi Qiu, Huichao Song, Jonah Bernhard, Steffen Bass, and Ulrich Heinz, "The iEBE-VISHNU code package for relativistic heavy-ion collisions," Comput. Phys. Commun. 199, 61-85 (2016), arXiv:1409.8164 [nucl-th]

[52] Z. Koba, Holger Bech Nielsen, and P. Olesen, "Scaling of multiplicity distributions in high-energy hadron collisions," Nucl.Phys. B40, 317-334 (1972)

[53] Aleksi Kurkela, Urs Achim Wiedemann, and Bin Wu, "Flow in AA and pA as an interplay of fluid-like and non-fluid like excitations," Eur. Phys. J. C79, 965 (2019), arXiv:1905.05139 [hep-ph]. 